\title{
Strength/duration relationships for electrocutaneous sensitivity: Stimulation by capacitive discharges
}

\author{
WILLARD D. LARKIN \\ University of Maryland, College Park, Maryland \\ and \\ J. PATRICK REILLY \\ Johns Hopkins University, Laurel, Maryland
}

\begin{abstract}
Methods of stimulating the skin by capacitive electrical discharges are described and properties of the stimulus waveforms are discussed. A neural excitation model is invoked to account for electrocutaneous sensitivity. The model gives a good account of threshold data from several experiments in which the methods of stimulation are compared. Sensitivity is shown to be chargedependent for stimuli that are brief relative to the exponential time constant of the neural membrane model. Systematic departures from charge-dependence occur when the stimulus discharge time constant is made to exceed a depolarization time constant: these departures follow a strength/duration relationship predicted by the model.
\end{abstract}

When the skin contacts an electrically charged object, and so provides a ground path for a capacitive discharge, a variety of cutaneous sensations are possible. Near threshold, the sensations commonly resemble touch or mild pinprick. Above threshold, the pinprick may be followed by a burning sensation akin to the delayed pain related to C-fiber activity. Bishop (1943) reported other sense qualities as well, and suggested that a punctate "spark" from a capacitor could be used selectively on the skin to excite discrete neural channels. If the difference of potential is great enough, a spark discharge may be seen and heard as well as felt.

To investigate the neural basis for transcutaneous electrical stimulation, it is useful to explore the sensory effects of variations in stimulus parameters. Much has been learned from such work (see, e.g., Babkoff, 1978; Crago, Peckham, Mortimer, Vander Meulen, 1974; Edelberg, 1971; Gibson, 1968; Girvin, Marks, Antunes, Quest, O'Keefe, Ning, \& Dobelle, 1982; Hahn, 1958; Pfeiffer, 1968; Rollman, 1974; Szeto \& Saunders, 1982; Tashiro \& Higashiyama, 1981). Nevertheless, a theoretical framework adequate to understand the various effects still is in need

We are grateful to V. T. Freeman, M. Flynn, L. B. Kittler, and R. J. Taylor for their many technical and intellectual contributions. This research was carried out at the Johns Hopkins University Applied Physics Laboratory, and was supported by the Canadian Electrical Association and the State of Maryland Department of Natural Resources. The opinions expressed herein are those of the authors, and are not necessarily endorsed by the project sponsors. The address for reprints is: W. Larkin, Department of Psychology, University of Maryland, College Park, MD 20742. of development. The results we report in this paper represent a facet of our effort in this direction. Our experiments focus on strength/duration relationships for human sensitivity to transcutaneous capacitive discharges. A variety of stimulating conditions are studied. We analyze the data from a neurological perspective and propose a general framework to account for the varied effects of stimulus parameters.

The capacitive discharge stimuli used in our experiments are monophasic (current flows in only one direction) and can be made brief in relation to the depolarization process in neural tissue. In these two respects, capacitive discharges resemble the shortduration, constant-current pulses often used in sensory and electrophysiological research (e.g., Bütikofer \& Lawrence, 1979; Gorman \& Mortimer, 1983; Pearce, Bourland, Neilsen, Geddes, \& Voelz, 1982; Sachs, Miller, \& Grant, 1980; Woolf, 1979) and in prosthetic devices (Hambrecht \& Reswick, 1977; Szeto \& Saunders, 1982). Accordingly, many of our conclusions can be applied to situations that use pulse waveforms different from ours, provided the stimuli are brief and monophasic.

In two other respects, our capacitive discharge stimuli differ from the current pulses commonly in use. First, the discharge waveform can have both a vary high initial voltage and a very high current peak, even at threshold levels of stimulation. Furthermore, while constant-current stimulation requires an electrode in good contact with the skin, a high-voltage capacitive discharge can occur without contact, if the electrode is simply brought close enough to sustain an electric arc between it and the skin. These charac- 
teristics of our stimuli are also present in electrostatic "carpet sparks" of the sort familiar to all who live in dry, upholstered environments (Chakravarti \& Pontrelli, 1976).

Adrian (1919), Bishop (1943), Bourguignon (1923), and others made good use of capacitor discharges in their pioneering explorations of skin sensation. Early work in electromyography also exploited the "punctate" quality of the capacitor discharge (Jones, 1913; Lapicque, 1938; Worster-Drought, 1920). The shift from this method to the modern reliance on constant-current devices stems mainly from a desire to compensate for instabilities in skin impedance (Tursky \& Watson, 1964). To achieve this goal, it is usually necessary to apply a low-resistance paste under the contact electrode, or even to abraid the corneal layer of skin (see Gibson, 1968). As we shall demonstrate, a strength/duration analysis of sensitivity to very brief capacitive discharges makes possible a different perspective on the role of skin impedance. We return to this issue in the Discussion section.

This paper reports on the determinants of threshold sensitivity for capacitive discharge stimulation. Section 1 describes the stimulus and its principal modes of application to the skin. Section 2 describes six experiments designed to elucidate the connection between skin sensitivity and neural excitability. To quantify this relationship, we invoke a neural excitation model. Equations used to derive the model have been presented in detail elsewhere (Reilly \& Larkin, 1983) and so will be summarized here in outline only.

\section{SECTION 1 \\ THE CAPACITIVE DISCHARGE STIMULUS}

\section{Apparatus and Methods}

A single capacitive discharge can be produced very simply by touching the skin with one side of a charged capacitor while the other side contacts a different point on the body. The stimulator we used for this purpose is described in Reilly and Larkin (1983; Reilly, Larkin, Taylor, \& Freeman, 1982). It uses a highvoltage source to charge a capacitor in either polarity, with a capability for potentials up to $15 \mathrm{kV}$. Capacitance, polarity, voltage, and discharge repetition are controllable. For the present report, only single capacitive discharges were used. The current path is through a large ground-return electrode, usually attached to the subject's forearm or leg. Electrode paste is used at the ground return, but not normally at the point of stimulation. The stimulus waveform can be captured by a wide-bandwidth current monitor (Pearson Model 150) and a high-voltage probe (Tektronix 6013A modified for 500-M $\Omega$ input), both of which connect to a digital storage oscilloscope (Nicolet 204A Explorer III). Subsequent waveform analysis makes use of a small computer (HewlettPackard HP 9826A).

A variety of stimulating electrodes were used. Those designed to be tapped with the fingertip were rigidly mounted on a housing unit (a sturdy plastic console box, approximately $23 \mathrm{~cm}$ square and $7 \mathrm{~cm}$ high). The tapped electrodes included a stainless steel sphere, $3.44 \mathrm{~cm}$ in diameter, and the rounded tip of a $1-\mathrm{mm}-$ diam brass rod which projected $0.5 \mathrm{~mm}$ from a 3.75 $\mathrm{cm}$-diam nylon housing. Other electrodes were fashioned as probes with insulated handles and small tips. These "pencil probes" were connected to the voltage source by a short, flexible cord, and could be used to touch small spots of skin at various points on the body. A "contact" method also was used: a cylindrical electrode rested continuously on the skin while its connecting cord was brought to a voltage source. The tapping, contact, and pencil probe arrangements are illustrated in Figure 1. In the contact condition, the spark gap occurs at the junction between two metal conductors, rather than between an electrode and the skin.

To determine thresholds, the subject adjusted the discharge voltage according to a strict protocol with paced trials (about 20 stimuli per minute), practiced stereotypy of movements-whether of the fingertip toward an electrode or of an electrode to a specified point on the skin-and controlled tapping force. Voltage was adjusted by a two-turn unmarked knob at

(a)
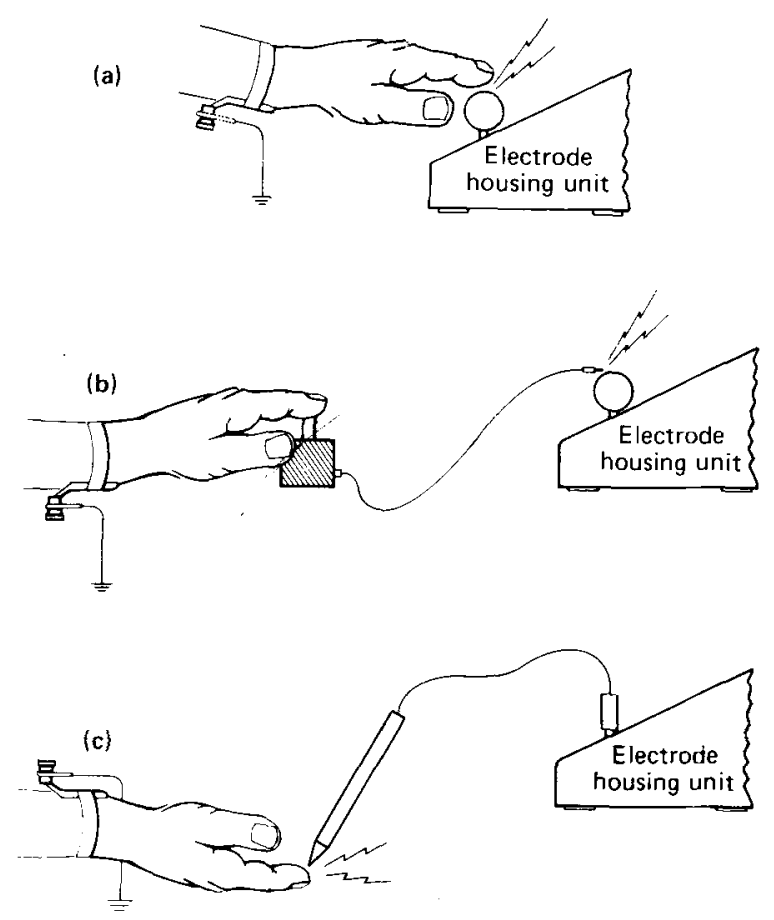

Figure 1. Three methods of applying capacitive discharges to the fingertip: (a) tapping an electrode, (b) using a contact electrode, and (c) touching the skin with a probe. 
the subject's testing booth. This knob acted as a vernier on a range control operated independently by the experimenter. Thus, in all tests the subject was blind to the voltage and rate-of-change of voltage resulting from the control knob manipulation.

The force of a tap on an electrode was registered by an accelerometer mounted inside the electrode housing unit. The accelerometer output was conveyed to a General Radio sound-level meter, Type 1933, which displayed the amplified signal on a Cweighted decibel scale. The subjects were trained to tap with a force that would produce excursions of the meter dial within $2 \mathrm{~dB}$ of a target position. A range of tap force from tactile threshold $(0 \mathrm{~dB})$ to tactile pain $(60 \mathrm{~dB})$ could be reliably produced. For the results in this paper, the electrode was tapped with the same "light" force, corresponding to $20 \pm 2 \mathrm{~dB}$ on the meter. Reilly et al. (1982) describe the calibration of tap force in absolute physical units.

Thresholds were measured in individual test sessions, each lasting 1 to $2 \mathrm{~h}$. Subjects adjusted the discharge voltage to a criterion of about $75 \%$ detection. Most subjects could adhere to this criterion without difficulty. Thresholds were checked occasionally with a "yes/no" forced-choice procedure, in which the voltage was held fixed, but was randomly on or off for each trial. By a "yes" or "no" response, the subject indicated whether a discharge was felt. Results of the forced-choice procedure were explained to the subjects, when necessary so that their detection criteria could be stabilized in the adjustment task.

The subject sat at a privacy booth in a quiet room, but could communicate freely with the experimenter. Apparatus sounds were masked by wideband noise continuously present in the subject's headphones. This masking noise also prevented aural detection of the stimuli. Visual detection was not a factor in these threshold experiments.

\section{Subjects}

The subjects were healthy adult volunteers. None had abnormal cardiovascular or dermatological conditions. Nineteen individuals served in various procedures, but not all are represented in every result to be reported. Each subject had had ample training and practice prior to formal testing.

\section{Stimulus Waveforms}

When a capacitor discharges through a resistance, both the current and the voltage have a simple exponential waveform. The rate of discharge can be succinctly described by the exponential time constant, $\tau=R C$, where $R$ is the resistance of the discharge path and $C$ is the capacitance. If the conductor is replaced by the human body, $R$ is no longer constant, but varies nonlinearly with the voltage on the capacitor. Nevertheless, as Figure 2 illustrates, the resulting waveform is still remarkably close to an exponen-

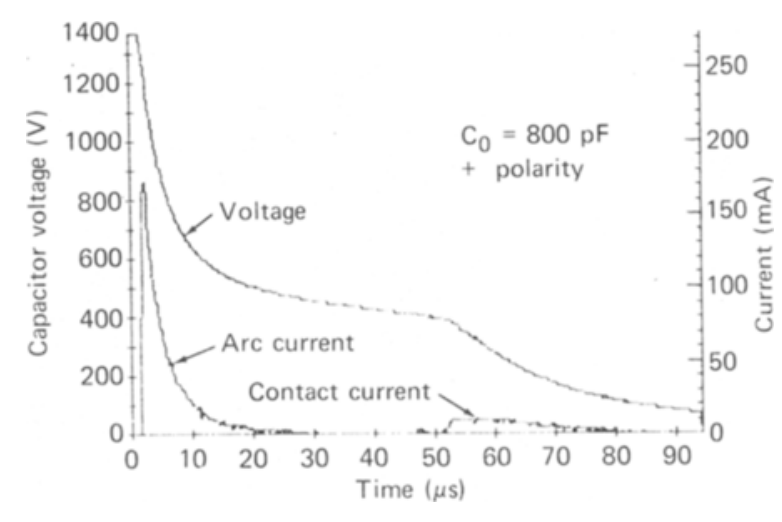

Figure 2. Example of a discharge waveform produced by tapping an electrode. Conditions of stimulation were chosen to display separate arc and contact components.

tial. To produce the example in Figure 2, an electrode was charged to $+1,400 \mathrm{~V}$ at a capacitance of $800 \mathrm{pF}$ and then discharged by a tap with the fingertip. During the first $25 \mu \mathrm{sec}$, the discharge was conveyed through an air gap, prior to mechanical contact. The arc current (lower trace) reached a peak of $175 \mathrm{~mA}$ and then decayed exponentially to zero, even though, as the upper trace reveals, the capacitor was not yet fully discharged. Mechanical contact occurred about $50 \mu \mathrm{sec}$ after the arc, initiating a second stage of discharge. The peak current flow in the contact stage was rather little compared with the arc current, and the rate of discharge of the waveform was slower in this portion than at the beginning. Between the two stages of discharge, the voltage reached a plateau of about $425 \mathrm{~V}$.

A voltage plateau and the resulting waveform segregation are characteristic features of capacitive discharges with a tapped electrode, although to our knowledge they have not been previously described. The plateau level depends on the initial voltage and on the thickness of the corneal layer. The plateau duration depends on the arc discharge rate and the approach velocity of the fingertip. Under some conditions (e.g., low initial voltage, slow arc discharge, and/or high approach velocity), the arc and contact stages can merge into a continuous, composite discharge. The current flow in the two stages is almost always unequally divided. At very low initial voltages, only contact current is seen. As the initial voltage on the capacitor is raised to the minimum required for an arc discharge, the transition from contact current to arc current is abrupt, and the arc component quickly becomes dominant above this point, with only residual current in the contact stage. These two stages of discharge are also observed when a capacitor is brought to a contact electrode (by the method of Figure $1 b$ ). In this mode of stimulation, discharges also have an exponential form, but with time constants that are inversely related to the area of skin contact, and with lower voltage plateaus. 
The arc discharge does not occur if the initial voltage is below the plateau level. On average, this level is about $550 \mathrm{~V}$ across subjects, capacitances, and other conditions of stimulation. Across these conditions, the plateau may range $150 \mathrm{~V}$ lower or higher, but it is always sharply defined for any specific situation. The absence of a low-voltage spark reflects, in part, the breakdown potential of air and the dielectric strength of the epidermis. Mason and Mackay (1976) excised a 15- $\mu$ layer of human corneal skin and found that it would sustain a potential difference of about $600 \mathrm{~V}$. This value is close to the plateau voltages we have measured.

All of the stimuli described in this paper have "positive polarity," that is, the stimulating electrode is anodal relative to the skin. The overall waveform pattern for negative-polarity stimulation is similar, but with occasional discontinuities in the arc discharge. While the positive-polarity current waveform is relatively smooth and repeatable, the negativepolarity version often has a bistable characteristic, with sudden transitions between two rates of discharge corresponding to two levels of impedance. Possible explanations for this saltatory behavior are discussed in Reilly and Larkin (1983).

Variations in waveform shape occur as a stimulus is moved from one point to another on the skin. Figure 3 illustrates this variation for four points $2 \mathrm{~mm}$ apart on a longitudinal axis of the fingertip. The voltage (upper trace) and current (lower trace) are shown. To produce these examples, a probe electrode (Figure 1c) was used in conjunction with a dielectric mask. A small puncture hole in the mask was positioned over desired points of stimulation. Only the arc component of the stimulus is shown in Figure 3. All four waveforms begin at $1,200 \mathrm{~V}$, and all have approximately exponential decays to a common plateau, but their rates of decay are different, possibly depending on local variations in skin impedance.

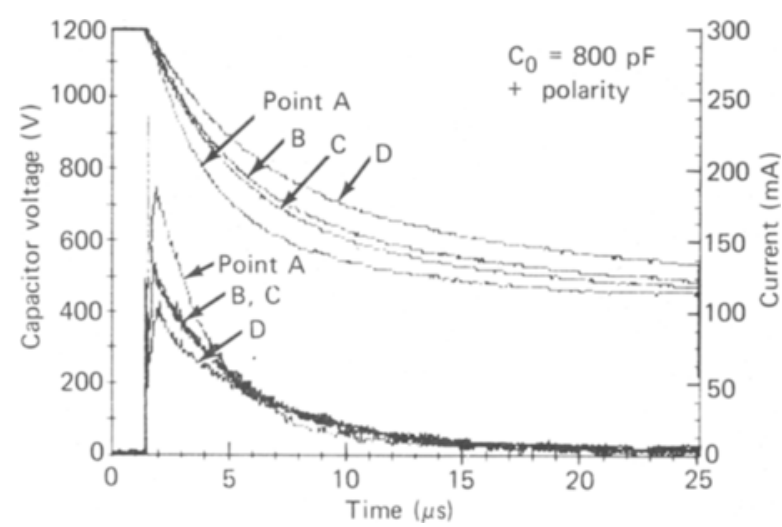

Figure 3. Voltage (upper) and current (lower) waveforms for stimulation at four different points on the fingertip: $2-\mathrm{mm}$ spacing between points.
In contrast, repeated stimuli at the same point produce nearly indistinguishable waveforms. We have found that the initial skin impedance can vary by as much as $2: 1$ among points only 1 or $2 \mathrm{~mm}$ apart. The arc plasma itself does not contribute significantly to the overall measured impedance, and so is not a factor in this variation (Reilly et al., 1982).

For the conditions studied in our experiments, an entire capacitive discharge usually is complete in less than $0.5 \mathrm{msec}$. Waveform variations within this time interval might therefore be thought irrelevant to perception: if the sensory system acts like a temporal integrator, the waveform fine-structure seems too rapid to be resolved. While this expectation is essentially correct, we show in Section 2 that the overall rate at which electrical charge passes into the skin, that is, the stimulus "time constant," is a parameter critical to sensation. We have measured stimulus time constants for a variety of conditions. Figure 4 illustrates a set of these measurements for a subject who tapped an electrode with the second digit of the left hand. (Data for a different subject are given in Reilly \& Larkin, 1983.) Each measurement was taken as the time, $\tau$, for the voltage over an exponential portion of the waveform to drop to $1 / \mathrm{e}$ of its initial value, relative to its terminal value. Because either the arc or the contact portion nearly always predominates, only one stage of each waveform is represented. Each plotted point represents an average of at least 12 waveform samples. When the voltage is low, the waveform is dominated by the contact stage of current flow, which tends to have a time constant in excess of $50 \mu \mathrm{sec}$. As the initial voltage is increased beyond the plateau level, the arc portion achieves sudden dominance and develops a much shorter time constant, dropping below $1 \mu \mathrm{sec}$ for some of the conditions in Figure 4. This rapid change in the discharge time constant reflects the nonlinear, voltage-dependent characteristic of skin impedance. In the next section, we discuss a neural hypothesis that relates cutaneous sensitivity to the stimulus time constant, and we present a variety of threshold data to test the hypothesis.

\section{SECTION 2 STRENGTH/DURATION THEORY AND DATA}

\section{Theory}

We wish to account for transcutaneous sensitivity in terms of the electrical response of neural elements. For the present, we assume that the site of neural excitation is axonal, although other structures may be involved as well. A circuit commonly invoked as an electrical analogue of the neural membrane is given in Figure 5 (after Aidley, 1971). Part a depicts a threenode section of myelinated nerve. Each node is represented by a parallel resistance-capacitance network. The ion-permeable portions of the membrane are represented by the transmembrane resistance, $R_{m}$, 


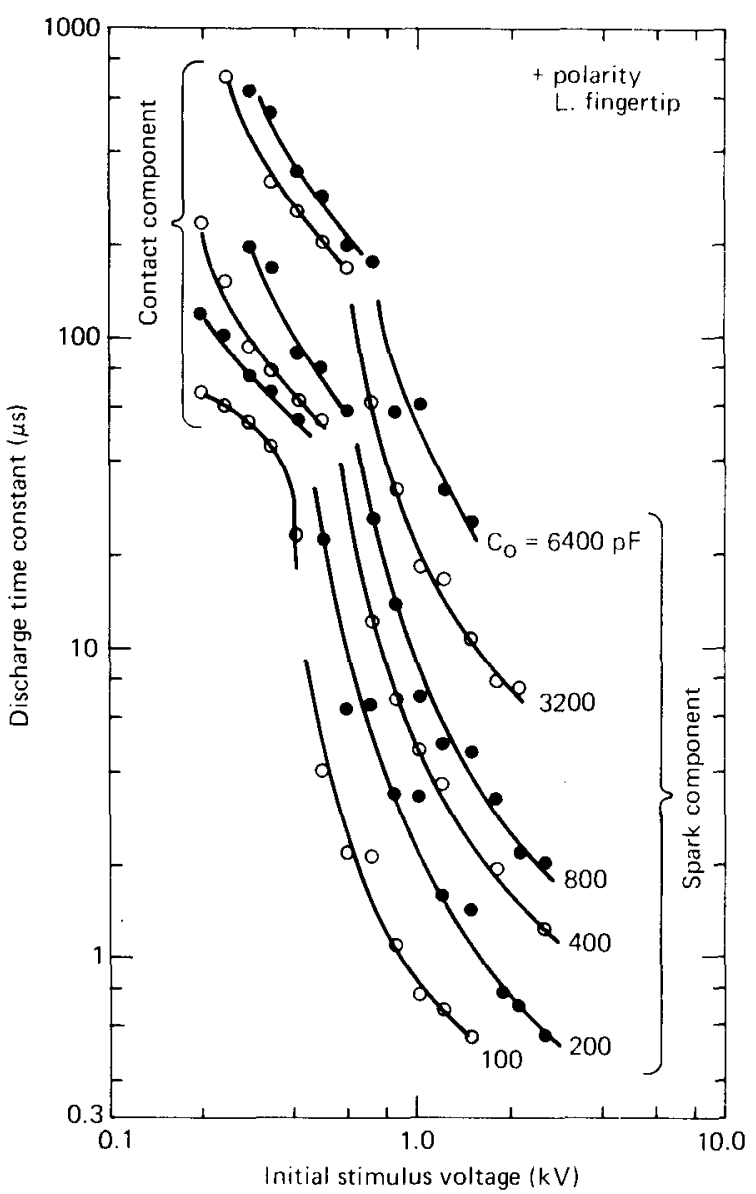

Figure 4. Time constants for capacitive discharge stimulation on the fingertip. Parameter shown is the stimulus capacitance, $\mathbf{C}_{\mathbf{0}}$.

and the impermeable portions by the transmembrane capacitance, $C_{m}$. The membrane resting potential is $E_{o}$, and $R_{a}$ is the node-to-node axoplasmic resistance. In part $b$, a single node is represented in the simplified form commonly used as an approximation to the spatially distributed model (see Koester, 1981; Woodbury, 1968). In this reduced version, $\mathrm{E}_{\mathrm{o}}$ is set to zero, intracellular resistance is ignored, and a driving current, $i(t)$, is indicated. When the voltage across the $\mathrm{RC}$ network rises to a threshold, the membrane is depolarized, and an action potential is produced. The rate of approach to threshold is exponential, with parameter $\tau_{m}$, which we refer to as the "membrane time constant." Although we have investigated model $\mathrm{a}$, the reduced version is sufficient for purposes of this paper. Some consequences of this simplification are mentioned in the Discussion.

We have analyzed the behavior of model $b$ for cases in which the current pulse is exponential:

$$
i(t)=I_{0} e^{-t / \tau}, \quad t \geqslant 0,
$$

where $I_{o}$ is the peak current and $\tau$ is the stimulus time constant. Although our capacitive discharge waveforms are not perfectly exponential (Section 1), they are nearly so, and there is little to be gained in going beyond the exponential approximation. The results of this analysis show that membrane excitability depends strongly on the stimulus time constant: for very short pulses, the action potential threshold follows a criterion of constant charge; for long pulses, it follows a criterion of constant current (Reilly \& Larkin, 1983). These tradeoffs are depicted in Figure 6. The horizontal axis plots the stimulus time constant, $\tau$, normalized by the membrane time constant, $\tau_{\mathrm{m}}$. When $\tau<<\tau_{\mathrm{m}}$, the charge required for depolarization is nearly constant. For $\tau>>\tau_{m}$, a constant, minimum current threshold is approached. For comparison, Figure 6 also displays the model's behavior in response to rectangular current pulses (Pearce et al., 1982). In the case of rectangular waveforms, $\tau$ should be interpreted as the pulse duration.

How well does the simple neuroelectric model account for human sensitivity? The observations of Rollman (1975) suggest that perceptual strength/ duration relationships may have their origin in neural excitability. By mapping out detectability curves for capacitive discharges varying in duration, we can provide quantitative tests of the model. In performing these tests, we use the single membrane model as if perceptual sensitivity required excitation in a single afferent neuron. The utility of this assumption will be evident in what follows, although we do not claim it as a substantive theory of perception.

\section{Experiments}

Six experiments were designed to elucidate strength/ duration tradeoffs for human sensitivity. In each experiment, the stimulus duration (discharge time constant) was manipulated and the effect on detection thresholds was observed. Stimulus discharge

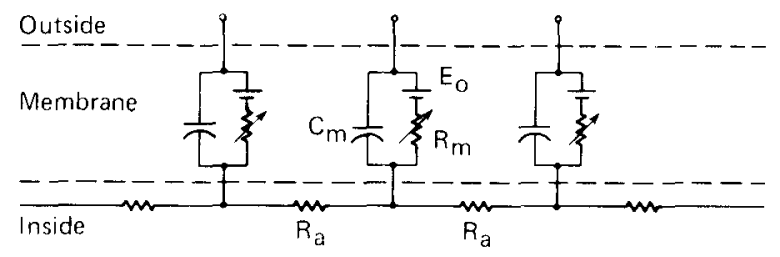

(a)

(b)

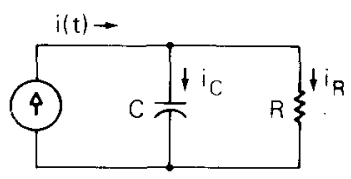

Figure 5. Electrical models for excitable membranes: (a) a threenode section of myelinated axon, and (b) a simplified linear circuit. 


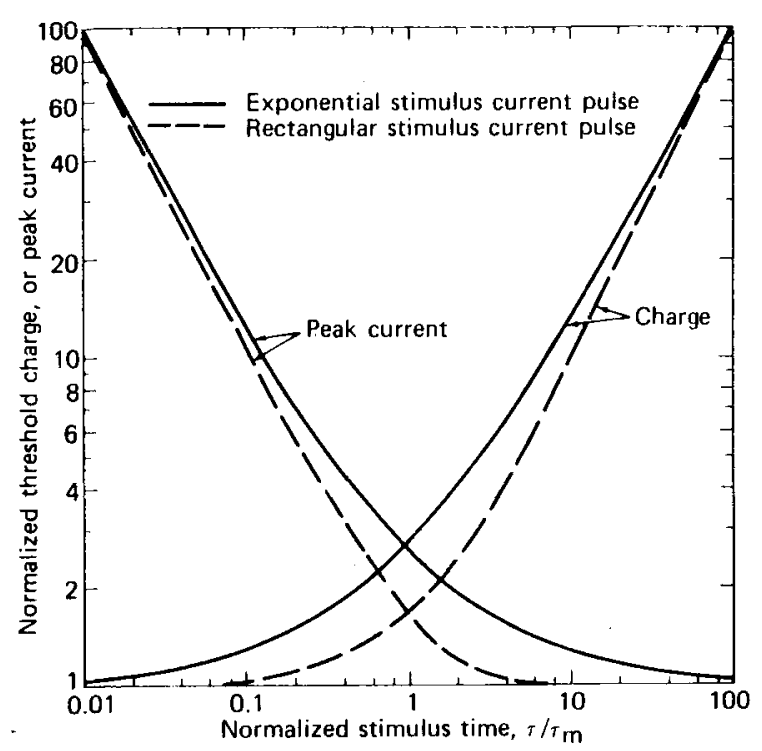

Figure 6. Normalized strength/duration curves for rectangular and exponential stimulus pulses.

time constants were manipulated by altering either the discharge capacitance (Experiments 1 through 4), the degree of skin hydration (Experiment 5), the resistance of the discharge circuit (Experiment 6), the size of a contact electrode (Experiments 2 and 3), or the mode of stimulation (a tapped electrode in Experiments 1 and 6; contact electrodes in Experiments 2, 3, and 5; subcutaneous electrodes in Experiment 4). These experimental conditions are summarized in Table 1 . In each case, the subjects adjusted the voltage on the capacitor to a threshold criterion, as described earlier in the Methods section.

When discharge capacitance was the experimental parameter, it ranged from 100 to $6,400 \mathrm{pF}$ in a geometric series and was changed randomly from one

Table 1

Summary of Experimental Conditions

\begin{tabular}{|c|c|c|c|c|}
\hline $\begin{array}{l}\text { Exp. } \\
\text { No. }\end{array}$ & $\begin{array}{c}\text { Mode of } \\
\text { Stimulation }\end{array}$ & $\begin{array}{c}\text { Experimental } \\
\text { Parameter }\end{array}$ & $\begin{array}{r}\text { Electrode } \\
\text { Type/Size }\end{array}$ & $\begin{array}{c}\text { No. of } \\
\text { Subjects }\end{array}$ \\
\hline 1 & $\begin{array}{l}\text { Tapped } \\
\text { Electrode }\end{array}$ & $\begin{array}{l}\text { Discharge } \\
\text { Capacitance }\end{array}$ & $\begin{array}{l}.01 \mathrm{~cm}^{2} \text { tip } \\
\text { in nylon } \\
\text { surround }\end{array}$ & 6 \\
\hline 2 & $\begin{array}{l}\text { Large contact } \\
\text { Electrode }\end{array}$ & $\begin{array}{l}\text { Discharge } \\
\text { Capacitance }\end{array}$ & $\begin{array}{l}1.27 \mathrm{~cm}^{2} \\
\text { circular } \\
\text { disk }\end{array}$ & 3 \\
\hline 3 & $\begin{array}{l}\text { Small contact } \\
\text { Electrode }\end{array}$ & $\begin{array}{l}\text { Discharge } \\
\text { Capacitance }\end{array}$ & $\begin{array}{l}.01 \mathrm{~cm}^{2} \\
\text { círcular } \\
\text { disk }\end{array}$ & 3 \\
\hline 4 & $\begin{array}{l}\text { Subcutaneous } \\
\text { Electrode }\end{array}$ & $\begin{array}{l}\text { Discharge } \\
\text { Capacitance }\end{array}$ & $\begin{array}{l}\text { Platinum- } \\
\text { coated } \\
\text { needles }\end{array}$ & 2 \\
\hline 5 & $\begin{array}{l}\text { Small contact } \\
\text { Electrode }\end{array}$ & $\begin{array}{l}\text { Dry vs. paste } \\
\text { treated skin }\end{array}$ & $\begin{array}{l}\text { Same as } \\
\text { Exp. } 3\end{array}$ & 3 \\
\hline 6 & $\begin{array}{l}\text { Tapped } \\
\text { Electrode }\end{array}$ & $\begin{array}{l}\text { Discharge cir- } \\
\text { cuit resistance }\end{array}$ & $\begin{array}{l}\text { Same as } \\
\text { Exp. } 1\end{array}$ & 7 \\
\hline
\end{tabular}

trial to the next without the subject's knowledge. In Experiment 1, subjects tapped a raised tip electrode with a constant, light tap force. In Experiments 2 and 3 , the fingertip rested on a cylindrical electrode, and a connecting wire periodically was brought into contact with the voltage source, as in Figure 1b. For the fourth experiment, thin platinum-coated needles were inserted in the volar forearm, penetrating to a depth of at least $0.5 \mathrm{~mm}$. Capacitive discharges were applied to the protruding ends of the needles by the method of Experiments 2 and 3. Because the needles pierce the corneal layer of skin, they bypass the principal site of electrical resistance. A penetration of $50 \mu \mathrm{m}$ on the forearm is sufficient to eliminate this high-impedance layer (Edelberg, 1971). The embedded needles caused very little skin injury and were not painful.

Figure 7 contrasts the results for the first four experiments. The geometric mean of the subjects' threshold voltage is plotted against discharge capacitance. Standard deviations of the individual threshold settings averaged about $12 \%$ of the mean in the tapping condition and about $5 \%$ of the mean in the contact and subcutaneous conditions. The latter value is consistent with the variability reported by Rollman (1969) for short square-wave pulses applied through contact electrodes. The extra variability in active tapping arises from interactions with tactile sensations and from heterogeneity in the locus of stimulation on the fingertip.

Individual differences in threshold sensitivity ranged up to $2: 1$ in voltage. These differences cannot entirely reflect response bias, inasmuch as all subjects adhered to the required detection criterion. The individual threshold contours were essentially parallel, and the data therefore could be combined by geometric averaging. Because our interest is in the shape of the detectability contours, not in absolute threshold values, the individual differences do not impair our analysis.

The threshold contours in Figure 7 take two distinct shapes: straight lines (representing a constant product of voltage, $V$, and capacitance, $C$ ) that have been drawn through the points representing the large contact electrode and the subcutaneous stimuli, and parallel curves of decreasing slope that have been drawn through the data for the tapped-electrode and the small contact electrode. The good fit of the straight lines indicates that sensitivity in two of the conditions is well described by a constant charge relationship (constant $\mathrm{Q}=\mathrm{CV}$ ). For the large contact electrode (contour $b$ in Figure 7), the minimum detectable charge is about $0.2 \mu \mathrm{C}-\mathrm{a}$ value consistent with results of other studies of brief transcutaneous pulses (Rollman, 1975). The distinctly lower thresholds for the subcutaneous stimuli may reflect an increase in charge density achieved by embedding the electrode in the skin, as well as the somewhat greater sensitivity of the forearm than of the fingertip. (Dif- 


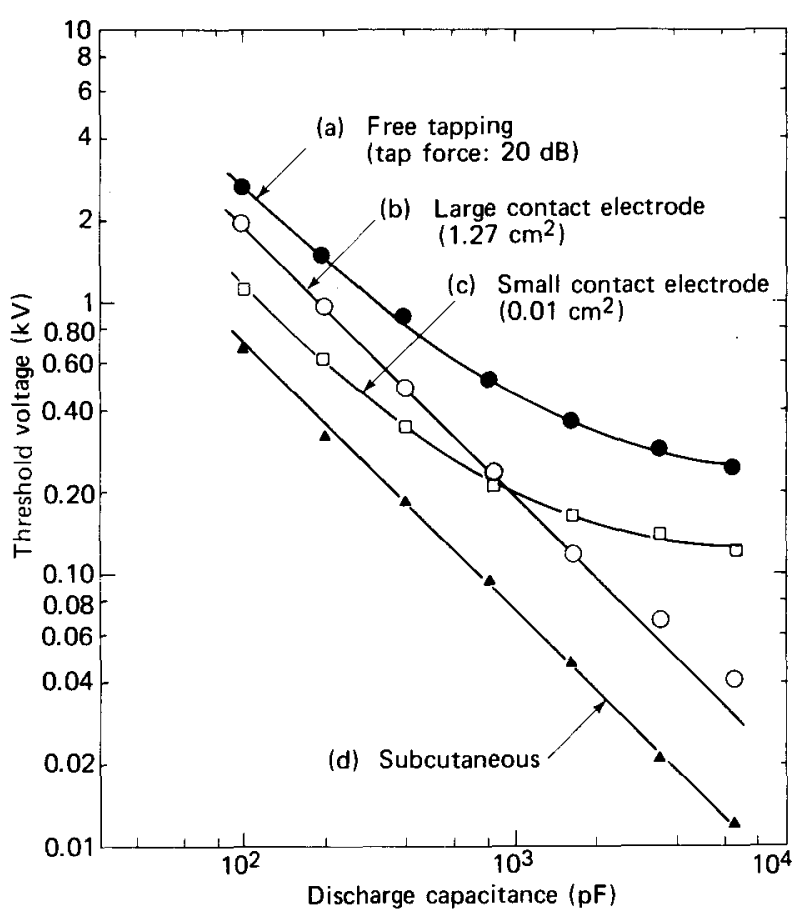

Figure 7. Sensitivity contours for four methods of stimulation: results of Experiments 1, 2, 3, and 4.

ferences in sensitivity among body loci were determined in separate experiments, and are discussed in Reilly et al., 1982.)

Thresholds for the tapped electrode and for the small contact electrode follow a constant charge relationship at low discharge capacitances, but deviate markedly from this relationship at high capacitances (see curves a and $\mathrm{c}$ in Figure 7). The detection threshold requires increasing levels of charge as the capacitance is raised. The vertical displacement of curve a relative to curve $c$ is largely accounted for by the masking effect of tactile stimulation (the finger tap). (Tactile masking was explored in a separate study; see Reilly \& Larkin, 1983.) Curve a appears to be immune to variations in the size and shape of the tapped electrode: whether subjects tapped a flat surface or a $3.4-\mathrm{cm}$-diam sphere, thresholds were virtually identical to those for the $1-\mathrm{mm}^{2}$ electrode plotted in Figure 7.

At low capacitance, current density considerations could be invoked to explain the relative positions of curves $\mathrm{b}$ and $\mathrm{c}$-the two contact electrode cases in Figure 7. For example, there is evidence that multiple conduction paths can operate in parallel when the electrode-to-skin interface is large. Using low-voltage $\mathrm{dc}$ pulses and a silver staining technique, Saunders (1974) counted about one current path per square millimeter. Accepting this value, our small electrode would qualify as a single-path device and our large electrode $\left(127 \mathrm{~mm}^{2}\right)$ could engage more than a hundred paths. But the lower current density with multi- path stimulation ought to make thresholds uniformly higher for the large electrode, not just at low capacitance. The crossover at high capacitance indicates that current density is not the only relevant parameter.

The contour shapes of Figure 7 can be accounted for by comparing stimulus time constants, $\tau$, for the various modes of stimulation. In curve $d$, the subcutaneous stimuli bypass the high-resistance layer of skin: these discharges have extremely short time constants, less than $1 \mu \mathrm{sec}$ for all of the data plotted. It is a safe assumption that neural membrane time constants, $\tau_{m}$, are at least an order of magnitude greater (cf. Aidley, 1971; Irnich, 1980; Ranck, 1975). Consequently, the entire subcutaneous curve lies in the constant charge region of the theoretical strength/ duration relationship (Figure 6), and the data have the linear profile and slope required by this relationship.

The stimulus time constants for the large contact electrode were nearly as brief, ranging from about $0.15 \mu \mathrm{sec}$ at $100 \mathrm{pF}$ to $3 \mu \mathrm{sec}$ at $6,400 \mathrm{pF}$. These values also place the stimuli in the constant charge portion of Figure 6. The straight line fit (curve $b$ in Figure 7) is good except at $6,400 \mathrm{pF}$, where the data tend in the direction that the neural model requires as $\tau$ approaches $\tau_{m}$.

For curves a and c, $\tau$ values reach $1 \mathrm{msec}$ or more at the high capacitances, so that greater charge is needed to bring the neural membrane network to its threshold. To examine the fit to the membrane model, stimulus time constants were measured for two subjects at their threshold points along curve $a$, and for one subject along curve $c$. Then the threshold level of charge at each point was expressed in proportion to the minimum detectable charge, estimated by the threshold at the lowest capacitance, $100 \mathrm{pF}$. A plot relating this normalized threshold to the stimulus time constant is given in Figure 8 (square and triangular points). Data are also shown for Experiment 6 , to be described below. The curves drawn through the plot are theoretical strength/duration relationships derived from the neural excitation model, using values of $\tau_{\mathrm{m}}$ from 0.2 to $1.0 \mathrm{msec}$. These assumed parameter values fall within the range of results reported by Bostock (1983), Heckmann (1972), Pfeiffer (1968), and Rollman (1975). There is scatter in the data, but the overall pattern is consistent with the model.

Differences in $\tau$ arise because the mode of application of the discharge stimulus governs the effective skin resistance. On dry skin, impedance declines (approximately as the $1 / 3$ power) of electrode contact area, so that the $1.27 \mathrm{~mm}^{2}$ electrode, and any larger one, will discharge the capacitor very rapidly in comparison with $\tau_{\mathrm{m}}$. Thus, thresholds for the large contact electrode are governed by a constant charge criterion, but thresholds for the small electrode depart from this criterion at high capacitance. 


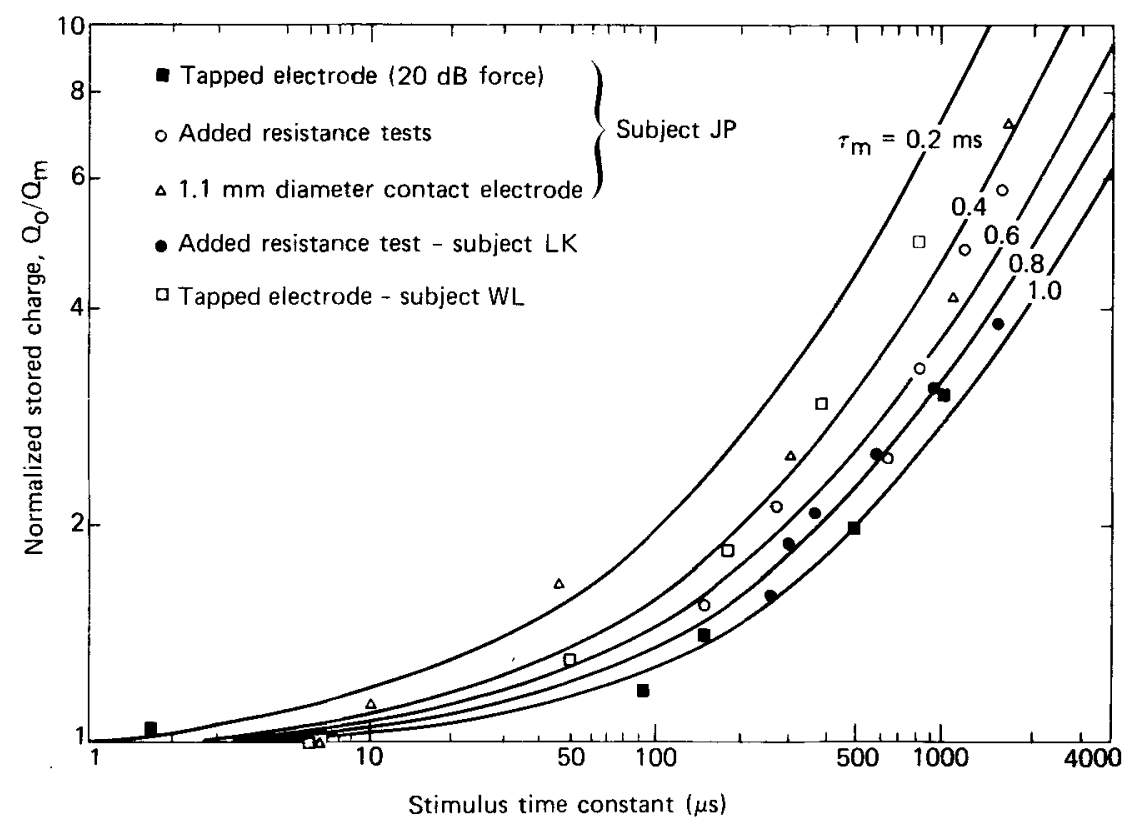

Figure 8. Strength/duration data. Points are measurements at detection threshold. Curves are theoretical relationships for exponential pulse stimulation, based upon assumed membrane time constants between 0.2 and 1.0 msec.

Two additional experiments were designed to manipulate stimulus time constants by altering the discharge conditions. In Experiment 5, skin resistance was reduced by rubbing electrode paste (Burdick Corp., "Liqui-Cor") into the fingertip. Using the same contact technique and $1-\mathrm{mm}^{2}$ electrode described for Experiment 3, thresholds were determined for three subjects. A no-paste condition was run in separate sessions. The principal effect of the paste treatment should be to lower the resistivity of the corneal layer of skin, and thus to shift stimulus time constants to values below those in Experiment 3. As a result, thresholds in the high-capacitance portion of curve $c$ should be reduced.

The two threshold contours for Experiment 5 are shown in Figure 9. In the no-paste condition, the data replicate curve $c$ of Figure 7. This curve has been replotted as the dashed line of Figure 9. When paste was applied, time constants were reduced and much of the curvature vanished, as shown by the solid line. The vertical separation of the two contours is an ancillary effect of the paste treatment. (We shall report separately on this and on related phenomena that appear to depend on spatial properties of the stimulus.) The main outcome of Experiment 5 is the paste-induced linearization of the threshold contour for a small contact electrode. Reductions in $\tau$ shift stimuli toward the constant-charge portion of the theoretical strength/duration curve (Figure 6). We interpret the linearization effect as a direct result of this shift, and as a confirmation of the model.
In the final procedure (Experiment 6), resistance was added in series with the discharge circuit. Resistor values were changed randomly from one trial to the next without the subject's knowledge. Four subjects were tested at fixed capacitances of 200 and $3,200 \mathrm{pF}$,

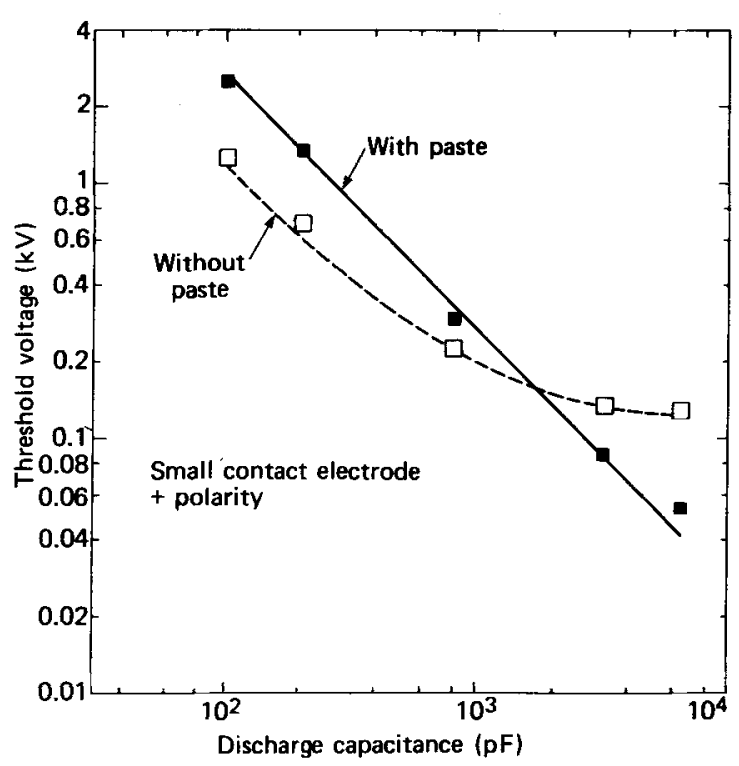

Figure 9. Effect of electrode paste on sensitivity, for $1-\mathrm{mm}^{2}$ contact on fingertip: results of Experiment 5. Data points are averaged thresholds for three subjects. Dashed line is an averaged threshold contour for five subjects, redrawn from Figure 7 (curve a). Solid line represents a constant charge (CV) relation. 
with added resistances of $0,40,80$, and $200 \mathrm{k} \Omega$. Three other subjects were tested at $800 \mathrm{pF}$, with the extra resistance extended to $1.6 \mathrm{M} \Omega$. In all other respects, the procedure was the same as for Experiment 1.

The added resistance increases the stimulus time constant, $\tau$. If $\tau$ increases substantially beyond the membrane parameter, $\tau_{\mathrm{m}}$, the neural excitation model predicts a loss of sensitivity. The results (Figure 10) show exactly this effect. Note that the threshold elevations are not substantial until the added resistance reaches about $10^{5} \Omega$. For the $800-\mathrm{pF}$ case, threshold doubling requires about $10^{6} \Omega$ of external resistance.

Samples of the discharge waveforms were recorded, and stimulus time constants were measured for two of the subjects along the 800-pF curve. Detection thresholds for these subjects, normalized with respect to their minimum charge thresholds, are plotted as the circular points in Figure 8. The data for Subject J.P. (open circles) approximate a neural strength/ duration curve with $\tau_{\mathrm{m}}=0.5 \mathrm{msec}$. For Subject L.K. (closed circles), the estimate of $\tau_{\mathrm{m}}$ is close to $0.8 \mathrm{msec}$. In both cases, the neural excitation model gives a good account of the data.

\section{Discussion}

This paper has described several techniques by which capacitive discharge stimulation can be applied to the skin. We have shown that a simple model of neural excitation accounts very well for the threshold data. The fit of the model depends on the relationship between two parameters: the time constant, $\tau$, of the capacitive discharge stimulus, and the empirically determined time constant, $\tau_{m}$, which characterizes the neural excitation response. If $\tau$ is small relative to $\tau_{\mathrm{m}}$, sensory thresholds are governed by a criterion of fixed charge. Departures from this criterion occur in some modes of stimulation because $\tau$ is sensitive to skin resistance. The degree of departure from charge-dependency is consistent with

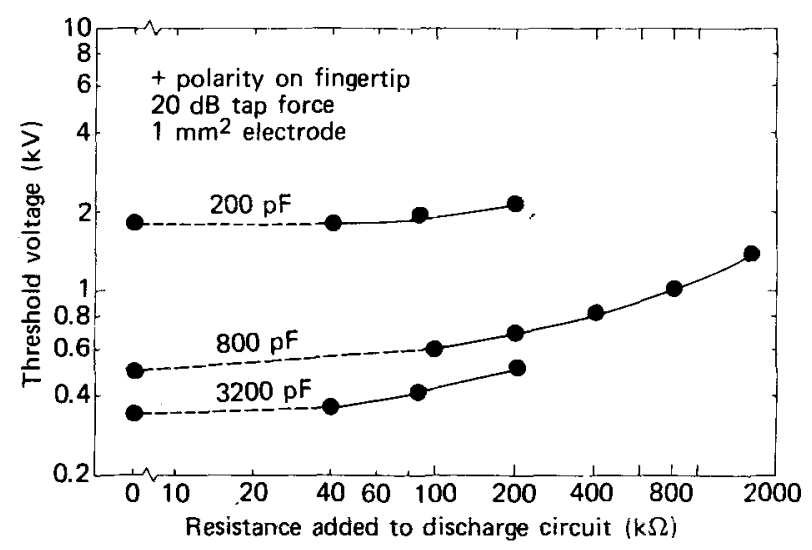

Figure 10. Effect of external resistance added to the discharge circuit: results of Experiment 6. Data are averaged thresholds for at least three subjects. strength/duration curves derived from the model, using assumed values of $\tau_{\mathrm{m}}$ between 0.2 and $1.0 \mathrm{msec}$. This range of inferred parameter values is consistent with data reported by others (Bostock, Sears, \& Sherratt, 1983; Heckmann, 1972; Rollman, 1975), although both smaller (Ranck, 1975) and larger (Pfeiffer, 1968) values have been found in stimulation of mammalian nerves.

The time constant governing a sensory strength/ duration curve does not necessarily provide an accurate measure of the membrane excitation process. To relate the electrocutaneous response to membrane properties may require a more refined model than the one used here. Among the refinements that could be made, three deserve immediate mention. First, an account is needed of the relationship between the waveform for a transcutaneous current pulse and that of the resulting current flow across a neural membrane. Our analysis has assumed these to be identical, although there are indications that the stimulus current waveform may not be the same as that crossing the membrane (McNeal, 1976). Second, we have treated the neuron as a linear system, ignoring the Frankenhauser-Huxley nonlinearities that more accurately describe membrane behavior (McNeal, 1976). Third, as there is always a spatial aspect in transcutaneous stimulation, a more refined approach would use a spatially extended model of the neuron (as in Figure 5a), rather than the simplified version we have used (Figure 5b).

Our preliminary work on these refinements (Reilly et al., 1983) indicates that the form of the strength/ duration curve with the simplified model is similar to that for more elaborate models. By restricting our stimuli to single monophasic discharges (current flowing in one direction), we avoid some of the analytical difficulties in more complex situations. However, the simplified model is not adequate in all cases. For example, it is clear that stimulation with biphasic current pulses does not follow the simple linear model (Reilly \& Larkin, 1984).

When spatial considerations are made explicit, as in model a of Figure 5, the theoretical strength/ duration curve has a form very close to that for the simpler model, but it can be shifted horizontally by an amount that depends on the ratio of intracellular to nodal resistance $\left(r_{a}: r_{m}\right)$. As a result, the apparent time constant governing sensory strength/duration data can be a biased estimate of $\tau_{\mathrm{m}}$. According to Bostock's (1983) computer simulation of myelinated nerve, a similar difficulty afflicts the interpretation of neural strength/duration data. Consequently, despite the good fit with neural data, our psychophysical results by themselves do not permit us to infer directly the temporal parameters of the membrane process. This limitation does not lessen the utility of the neural model as a framework for understanding psychophysical relationships. Estimated values of 
$\tau_{m}$, which we have regarded as "apparent membrane time constants," provide an empirical, quantitative basis for predicting cutaneous sensitivity.

Bishop's (1943) original experiments with capacitive discharges made good use of the fact that highvoltage sparks can arc from the electrode to the skin, avoiding any mechanical contact. He reported that there were both intensive and qualitative variations in sensory effects among closely spaced sites of stimulation. Although our experiments were not designed to check Bishop's findings, we observed similar pointto-point variations in sensitivity. Moreover, we found that experienced subjects could discriminate among two stimuli at the same site if the stimulus time constants were very different. Typically, a rapid discharge (e.g., one from a 100-pF capacitor) evokes a "touch" or mild "pinprick" sensation at nearthreshold levels, whereas a slow discharge (e.g., one from a $6,400-\mathrm{pF}$ capacitor) can also produce a delayed "burning" sensation. These effects indicate that the electrical discharge may be "tunable," in some degree, to the temporal excitation requirements of different classes of cutaneous neurons. Gorman and Mortimer (1983) have reported such a selective effect in direct neural stimulation. The mechanism for this selectivity is not yet understood. It is likely, however, that our threshold contours (Figure 7) reflect the mixed participation of different neural populations. To the extent that these fibers have differing rates of membrane depolarization, the model parameter, $\tau_{m}$, should be regarded as a sample average of neural time constants, rather than as a fixed number. We are not aware of any data linking membrane time constants to the major subclasses of sensory neurons. In view of the descriptive success of the membrane excitation model, such data could be a valuable source of new insight.

A major conclusion from the present work is that sensitivity to very brief electrocutaneous pulses is governed by a different criterion than is sensitivity to long pulses. The sensory system appears to scale short-duration stimuli in units of charge, and longduration stimuli in units of current. This observation was, of course, implicit in much early work on neural excitation, and it is consistent with prior studies of electrocutaneous sensation (e.g., Girvin et al., 1982; Pfeiffer, 1968; Rollman, 1974). Our analysis of strength/duration results does not fix a sharp boundary between "long" and "short," but indicates that for sensory effects to be charge-dependent, the stimulus must be at least as brief as the presumed temporal requirements of neural depolarization. With capacitive discharge stimulation, the duration of the stimulus depends on a variety of factors other than the capacitance itself. Many of these factors, for example, degree of skin hydration or size of contact electrode, can be experimentally controlled in order to optimize conditions for charge-dependency. The present results provide some practical guidelines in this respect. For example, it is clear that unless the capacitance is very large, any discharge applied by means of a moderately sized contact electrode will have charge-dependent effects (curve $b$ in Figure 7), whereas a small electrode under the same conditions cannot produce a sufficiently rapid stimulus to achieve charge-dependency, except at very small capacitances (curve $c$ in Figure 7).

The difference between effects with small and large electrodes stems from the inverse relationship between electrode size and the resistance of the discharge circuit. Because the rapidity of a capacitive discharge depends on this resistance, any factor that affects resistance also affects the degree to which sensitivity will be charge-dependent. As we have shown, however, the thresholds in all of our conditions converged toward a constant charge criterion as the capacitance was decreased. Thus, it is possible to circumvent variations in skin resistance simply by ensuring that the stimulus pulse is sufficiently brief: by choosing a capacitance no larger than about $200 \mathrm{pF}$, one can be reasonably certain that the sensory effect will be governed by the amount of charge delivered to the epidermis, regardless of the mode of stimulation.

The importance of temporal factors in neural excitability has long been known (see Rushton, 1935), and is the basis of routine electrodiagnosis in clinical neurology (see Licht, 1971). Yet, we are not aware of any previous attempt to forge a quantitative link between cutaneous sensation and the behavior of excitable cells. The present paper represents a beginning in this direction. To be sure, the neural excitation model we have used can be regarded only as a rough approximation to biophysical reality. Nevertheless, it provides a framework within which the effects of varying stimulus conditions can be predicted and understood.

\section{REFERENCES}

Adrian, E. D. (1919). The response of human sensory nerves to currents of short duration. Journal of Physiology (London), $53,70-85$.

AIDLEY, D. J. (1971). The physiology of excitable cells. Cambridge: Cambridge University Press.

BАвкоFF, H. (1978). Electrocutaneous psychophysical inputoutput functions and temporal integration. Perception \& Psychophysics, 23, 251-257.

Bishop, G. H. (1943). Responses to electrical stimulation of single sensory units of the skin. Journal of Neurophysiology, 6, 361-382.

Bostock, H. (1983). The strength-duration relationship for excitation of myelinated nerve: Computed dependence on membrane parameters. Journal of Physiology (London), 341, 59-74.

Bostock, H., Sears, T. A., \& Sherratt, R. M. (1983). The spatial distribution of excitability and membrane current in normal and demyelinated mammalian nerve fibers. Journal of Physiology (London), 341, 41-58.

Bourguignon, G. (1923). La chronaxie chez l'homme. Paris: Masson. 
Bütikofe R, R., \& Lawrence, P. D. (1979). Electrocutaneous nerve stimulation-II: Stimulus waveform selection. IEEE Transactions: Biomedical Engineering, BME-26, 69-75.

Chakravarti, K., \& Pontrelli, G. J. (1976). The measurement of carpet static. Textile Research Journal, 46, 129-134.

Crago, P. E., Peckham, P. H., Mortimer, J. T., \& Vander Meulen, J. P. (1974). The choice of pulse duration for chronic electrical stimulation via surface, nerve, and intramuscular electrodes. Annals of Biomedical Engineering, 2, 252-264.

Edelberg, R. (1971). Electrical properties of the skin. In H. R. Elden (Ed.), Biophysical properties of the skin. New York: Wiley-Interscience.

GibSON, R. H. (1968). Electrical stimulation of pain and touch. In D. R. Kenshalo (Ed.), The skin senses. Springfield, IL: Thomas.

Girvin, J. P., Marks, L. E., Antunes, J. L., Quest, D. O., O'Keefe, M. D., Ning, P., \& Dobelle, W. H. (1982). Electrocutaneous stimulation $\mathrm{I}$. The effects of stimulus parameters on absolute threshold. Perception \& Psychophysics, 32, 524-528.

Gorman, P. H., \& Mortimer, J. T. (1983). The effect of stimulus parameters on the recruitment characteristics of direct nerve stimulation. IEEE Transactions: Biomedical Engineering, $B M E-30,407-414$

HAHN, J. F. (1958). Cutaneous vibratory thresholds for squarewave electrical pulses. Science, 127, 879-880.

Hambrecht, F. T., \& Reswick, J. B. (Eds.). (1977). Functional electrical stimulation: Applications in neural prostheses. New York: Marcel Dekker.

HeckmanN, J. R. (1972). Excitability curve: A new technique for assessing human peripheral nerve in vivo, Neurology, 22, 224-230.

IRNICH, W. (1980). The chronaxie time and its practical importance. $P A C E, 3,292-301$.

JoNEs, H. L. (1913). The use of condenser discharges in electrical testing. Proceedings of the Royal Society of Medicine, 42, 49-61.

Koester, J. (1981). Functional consequences of passive electrical properties of the neuron. In E. R. Kandel \& J. H. Schwartz (Eds.), Principles of neural science. New York: Elsevier/NorthHolland.

LAPICQUE, L. (1938). La chronaxie et ses applications physiologiques. Paris: Hermann \& Cie.

Licht, S. H. (Ed.). (1971). Electrodiagnosis and electromyography. New Haven: Licht.

Mason, J. L., \& Mackay, N. A. M. (1976). Pain sensations associated with electrocutaneous stimulation. IEEE Transactions: Biomedical Engineering, BME-23, 405-409.

MCNEAL, D. R. (1976). Analysis of a model for excitation of myelinated nerve. IEEE Transactions: Biomedical Engineering, $B M E-23,329-337$.

Pearce, J. A., Bourland, J. D., Neilsen, W., Geddes, L. A. \& Voelz, M. (1982). Myocardial stimulation with ultrashort duration current pulses. $P A C E, 5,52-58$.

Pfeiffer, E. A. (1968). Electrical stimulation of sensory nerves with skin electrodes for research, diagnosis, communication, and behavioral conditioning: A survey. Medical and Biological Engineering, 6, 637-651.

RANCK, J. B. (1975). Which elements are excited in electrical stimulation of mammalian central nervous system: A review. Brain Research, 98, 417-440.
REILly, J. P., \& LaRKiN, W. D. (1983). Electrocutaneous stimulation with high-voltage capacitive discharges. IEEE Transactions: Biomedical Engineering, BME-30, 631-641.

REILly, J. P., \& LARKIN, W. D. (1984). Mechanisms for human sensitivity to transient electric currents. In Proceedings of the Symposium on Electrical Shock Safety Criteria (The Electric Power Research Institute and the Canadian Electrical Association). Elmsford, NY: Pergamon Press.

Reilly, J. P., Larkin, W. D., Taylor, R. J., \& Freeman, V. T. (1982). Human reactions to transient electrical currents (Report No. CPE-8203). Laurel, MD: The Johns Hopkins University Applied Physics Laboratory. (NTIS No. PB83-204628).

Reilly, J. P., Larkin, W. D., Taylor, R. J., Freeman, V. T., \& KitTle R, L. B. (1983). Human reactions to transient electrical currents (Report CPE-8305). Laurel, MD: The Johns Hopkins University Applied Physics Laboratory. (NTIS No. PB84-112895).

Rollman, G. B. (1969). Electrocutaneous stimulation: Psychometric functions and temporal integration. Perception \& Psychophysics, 5, 289-293.

Rollman, G. B. (1974). Electrocutaneous stimulation. In F. A. Geldard (Ed.), Conference on Cutaneous Communication Systems and Devices. Austin, TX: Psychonomic Society.

Rollman, G. B. (1975). Behavioral assessment of peripheral nerve function. Neurology, 25, 339-342.

Rushton, W. A. H. (1935). The time factor in electrical excitability. Biological Reviews, 10, 1-17.

Sachs, R. M., Mille r, J. D., \& Grant, K. W. (1980). Perceived magnitude of multiple electrocutaneous pulses. Perception \& Psychophysics, 28, 255-262.

Saunders, F. A. (1974). Electrocutaneous displays. In F. A. Geldard (Ed.), Conference on Cutaneous Communication Systems and Devices. Austin, TX: Psychonomic Society.

Szeto, A. Y. J., \& Saunders, F. A. (1982). Electrocutaneous stimulation for sensory communication in rehabilitation engineering. IEEE Transactions on Biomedical Engineering, $B M E-29,300-308$.

Tashiro, T., \& Higashiyama, A. (1981). The perceptual properties of electrocutaneous stimulation: Sensory quality, subjective intensity, and intensity-duration relation. Perception \& Psychophysics, 30, 579-586.

Tursky, B., \& Watson, P. D. (1964). Controlled physical and subjective intensities of electric shock. Psychophysiology, 1, 151-162.

Woodbury, J. W. (1968). Action potential: Properties of excitable membranes. In T. C. Ruch, H. D. Patton, J. W. Woodbury, \& A. L. Towe (Eds.), Neurophysiology (2nd ed.). Philadelphia: Saunders.

Woolf, C. J. (1979). Transcutaneous electrical nerve stimulation and the reaction to experimental pain in human subjects. Pain, 7, 115-127.

Worster-Drought, C. (1920). Condenser tests in electrical diagnosis and prognosis of nerve injuries. British Medical Journal, 2, 389-392

(Manuscript received December 9, 1983; revision accepted for publication April 26, 1984.) 